\title{
The Hedgehog Signaling Networks in Lung Cancer: The Mechanisms and Roles in Tumor Progression and Implications for Cancer Therapy
}

\author{
Yoshinori Abe and Nobuyuki Tanaka \\ Department of Molecular Oncology, Institute for Advanced Medical Sciences, Nippon Medical School, 1-396 Kosugi-cho, \\ Nakahara-ku, Kawasaki 211-8533, Japan \\ Correspondence should be addressed to Nobuyuki Tanaka; nobuta@nms.ac.jp
}

Received 7 September 2016; Accepted 23 November 2016

Academic Editor: Daisuke Miyoshi

Copyright (C) 2016 Y. Abe and N. Tanaka. This is an open access article distributed under the Creative Commons Attribution License, which permits unrestricted use, distribution, and reproduction in any medium, provided the original work is properly cited.

Lung cancer is the most common cause of cancer-related death worldwide and is classified into small cell lung cancer (SCLC) and non-small-cell lung cancer (NSCLC). Several gene mutations that contribute to aberrant cell proliferation have been identified in lung adenocarcinoma, a part of NSCLC. Various anticancer drugs that target these mutated molecules have been developed for NSCLC treatment. However, although molecularly targeted drugs are initially effective for patients, the 5-year survival rate remains low because of tumor relapse. Therefore, more effective drugs for lung cancer treatment should be developed. The hedgehog (HH) signaling pathway contributes to organ development and stem cell maintenance, and aberrant activation of this signaling pathway is observed in various cancers including lung cancer. In lung cancer, $\mathrm{HH}$ signaling pathway upregulates cancer cell proliferation and maintains cancer stem cells as well as cancer-associated fibroblasts (CAFs). Furthermore, physical contact between CAFs and NSCLC cells induces HH signaling pathway activation in NSCLC cells to enhance their metastatic potential. Therefore, HH signaling pathway inhibitors could be a useful option for lung cancer therapy.

\section{Introduction}

Lung cancer is a leading cause of cancer-related death worldwide [1]. Lung cancer is classified into two major types: small cell lung cancer (SCLC) and non-small-cell lung cancer (NSCLC) (Figure 1). SCLC arises in the midlevel airway and is a very aggressive, highly metastasizing and lethal cancer type that comprises $15 \%$ of all lung cancers. NSCLC is the major type of lung cancer and comprises $85 \%$ of all lung cancers. NSCLC includes lung adenocarcinoma, lung squamous cell carcinoma (LSCC), and lung large cell carcinoma. Adenocarcinoma arises in the distal airway and its incidence is not related to smoking. LSCC arises in the proximal airway and is more aggressively and strongly associated with smoking than adenocarcinoma. Large cell carcinoma arises in the distal airway and the cancer cell mass is larger than the other two types of NSCLC. Large cell carcinoma is also an aggressive tumor [2]. Despite our current understanding of lung cancer, the precise molecular mechanisms underlying tumorigenesis in the lung have still not been completely determined.

Several signaling pathways are aberrantly activated in lung cancer cells. Key oncogenic mutations, so-called driver mutations, in components of these signaling pathways have been identified in lung adenocarcinoma. These include epidermal growth factor receptor (EGFR), the Kirsten rat sarcoma viral oncogene homolog GTPase (KRAS), a member of the rapidly accelerated fibrosarcoma (RAF) family, B-RAF $(B R A F)$, and the fusion oncogene echinoderm microtubuleassociated protein-like 4-anaplastic lymphoma receptor tyrosine kinase (EML4-ALK) [3, 4]. Furthermore, gene amplifications of avian erythroblastic leukemia viral oncogene homolog 2 (ERBB2), MET, ROS1, Neuregulin 1 (NRG1), neurotrophic tyrosine kinase receptor 1 (NTRK1), and RET have also been found in lung adenocarcinoma [5-8]. In LSCC, discoidin domain-containing receptor 2 (DDR2), fibroblast growth factor receptor 1 (FGFR1), FGFR2, and FGFR3 and 


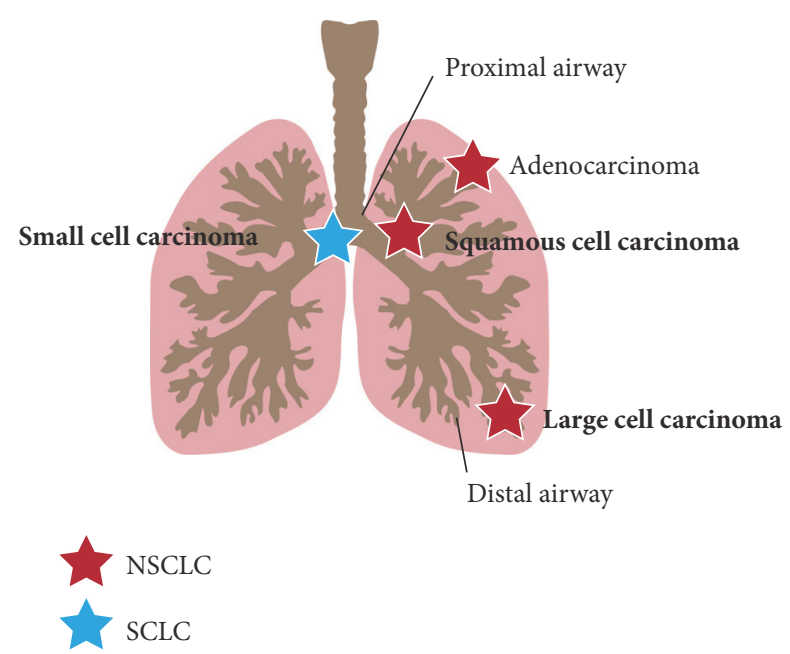

FIgURE 1: Lung cancer. Lung cancer is mainly classified into small cell lung cancer (SCLC) and non-small-cell lung cancer (NSCLC). NSCLC is further classified into adenocarcinoma, squamous cell carcinoma, and large cell carcinoma. Adenocarcinoma is the most common lung cancer and arises in the distal airway. Squamous cell carcinoma and SCLC arise in the proximal airway. Large cell carcinoma also arises in the distal airway.

genes in the phosphatidylinositol 3-kinase (PI3K) pathway seem to be more commonly mutated [9]. These gene mutations and gene amplifications induce activation of signaling pathways related to cell proliferation, such as the Rasextracellular signal-regulated kinase (ERK) pathway and the signal transducer and activator of transcription 3 (STAT3) pathway. NSCLCs harboring EGFR mutations or ALK gene rearrangements have been successfully targeted with tyrosine kinase inhibitors (TKIs) [10, 11]. However, these TKIs have not yet been shown to improve the overall survival in patients because of tumor recurrence [12]. Moreover, there are no effective drugs for SCLC, LSCC, and large cell carcinoma. Therefore, the 5 -year survival rate of lung cancer is only $16 \%$ at present [1].

A number of morphogenic signaling pathways that regulate developmental processes and organ homeostasis play critical roles in lung tumorigenesis. Studies of cancer stem cells (CSCs) support the idea that tumors harbor hallmarks of early development in their gene expression repertoire [13]. Recently, remarkable findings from an early stage clinical trial of an inhibitor for the hedgehog $(\mathrm{HH})$ signaling pathway have renewed hope that disruption of developmental signaling in tumors can be of therapeutic benefit $[14,15]$. HH pathway inhibitors block both intrinsic signaling in cancer cells and extrinsic signaling to stromal cells to reduce tumor growth [16]. These two strategies exploit distinct oncogenic functions of the pathway. As the $\mathrm{HH}$ signaling pathway is activated in SCLC as well as NSCLC, HH pathway inhibitors are expected to become a useful tool for treatment of lung cancer.

In this review, we discuss the roles of the $\mathrm{HH}$ signaling pathway in tumor development in SCLC and NSCLC and components of the $\mathrm{HH}$ signaling pathway that represent viable lung cancer therapy targets.

\section{The HH Signaling Pathway}

The HH signaling pathway regulates morphogenesis of various organs during embryogenesis [17]. The $\mathrm{HH}$ signaling pathway also regulates stem cell renewal and organ homeostasis in the adult [18]. The molecular mechanisms of the $\mathrm{HH}$ pathway are complex, and several comprehensive reviews have been published describing the detailed mechanisms [1921]. In the canonical $\mathrm{HH}$ signaling pathway, three $\mathrm{HH}$ ligands have been identified: Sonic Hedgehog (SHH), Indian Hedgehog (IHH), and Desert Hedgehog (DHH). Each HH ligand has distinct spatial and temporal expression patterns and activates $\mathrm{HH}$ signaling by binding to Patched (PTCH), a 12-pass transmembrane-spanning receptor. In the absence of $\mathrm{HH}$ ligand, PTCH is localized to primary cilia and constitutively suppresses the activity of Smoothened (SMO), a 7-pass transmembrane-spanning protein, which is a member of the Gprotein-coupled receptor superfamily [22] (Figure 2). In addition to $\mathrm{PTCH}$, additional $\mathrm{HH}$ ligands binding cell surface proteins, such as CAM-related/downregulated by oncogenes (CDO), brother of Cdo (BOC), and growth-arrest-specific 1 (GAS1), have been identified, and these molecules function as $\mathrm{HH}$ ligand coreceptors to facilitate $\mathrm{HH}$ signal reception $[23,24]$. Following binding of one of the three $\mathrm{HH}$ ligands to PTCH, SMO accumulates in the primary cilia and facilitates the activation of GLI transcriptional activators and their translocation into the nucleus to activate expression of $\mathrm{HH}$ target genes, including GLI1 and PTCH genes (Figure 2) [25, 26]. Suppressor of fused (SUFU) is a key negative regulator of the $\mathrm{HH}$ signaling pathway [27]. In the absence of $\mathrm{HH}$ ligands, SUFU inhibits $\mathrm{HH}$ signaling by sequestration of GLI proteins in the cytoplasm and by promoting the formation of the GLI3 repressor (GLI3R). A nuclear function for SUFU in chromatin has also been suggested.

In vertebrates, the GLI family consists of three proteins, GLI1, GLI2, and GLI3 [21]. All GLI proteins contain an activator domain (GLI-A) at their C-terminus; GLI2 and GLI3 also have an N-terminal repressor domain (GLI-R) [28]. Studies in mutant mice suggest that GLI2 is the major activator of $\mathrm{HH}$ signaling pathway [29], whereas GLI3 is the major repressor $[30,31]$. GLI1 most likely serves as a signal amplifier downstream of GLI2 [29, 32]. Gli2 knockout (KO) mice die at birth, whereas Gli1 KO mice show normal development, unless one copy of Gli2 is also defective [33]. Interestingly, experiments in mutant mice further suggest that GLI2 can rescue GLI1 protein function, whereas Gli1 knock-in into the Gli2 allele can rescue the Gli2 null phenotype [34]. Upon binding of the $\mathrm{HH}$ ligand to the receptor PTCH, followed by SMO activation, SUFU-GLI2 and SUFU-GLI3 complexes dissociate and GLI2 and GLI3 translocate into the nucleus, where they activate expression of $\mathrm{HH}$ target genes, including GLI1 and PTCH [35]. The balance between the activating and repressive forms of the GLI family transcription factors results in the expression of target genes [21].

The HH signaling pathway has critical roles during embryonic lung development as well as postnatal lung development [36]. During embryonic lung development, $\mathrm{HH}$ signaling pathway molecules dramatically change expression patterns and expression levels. The SHH expression pattern 


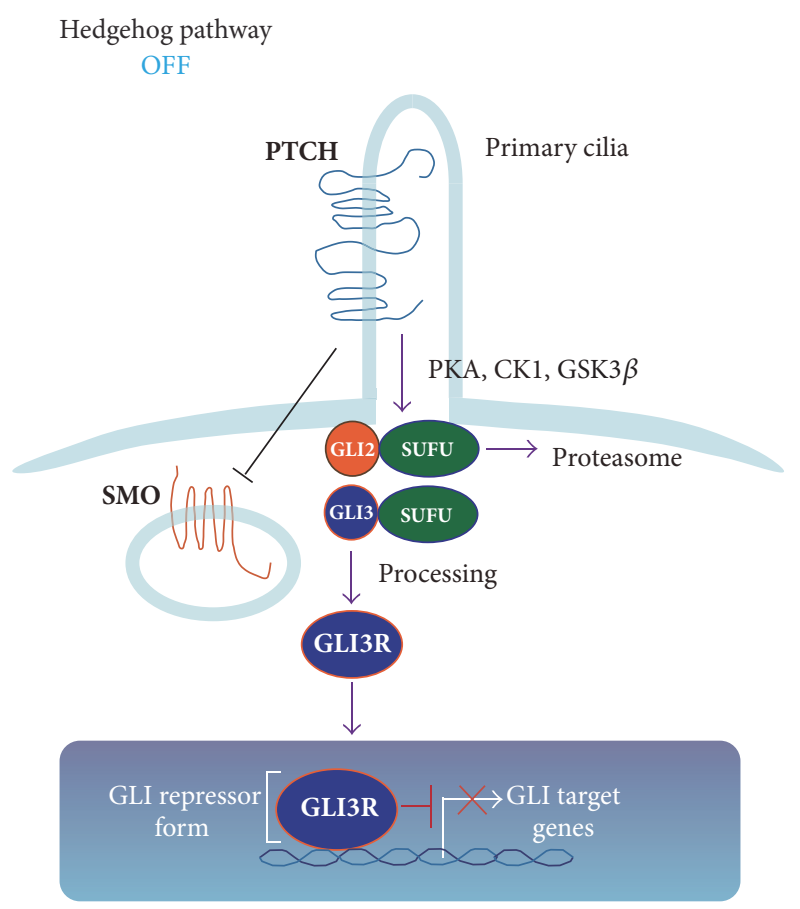

(a)

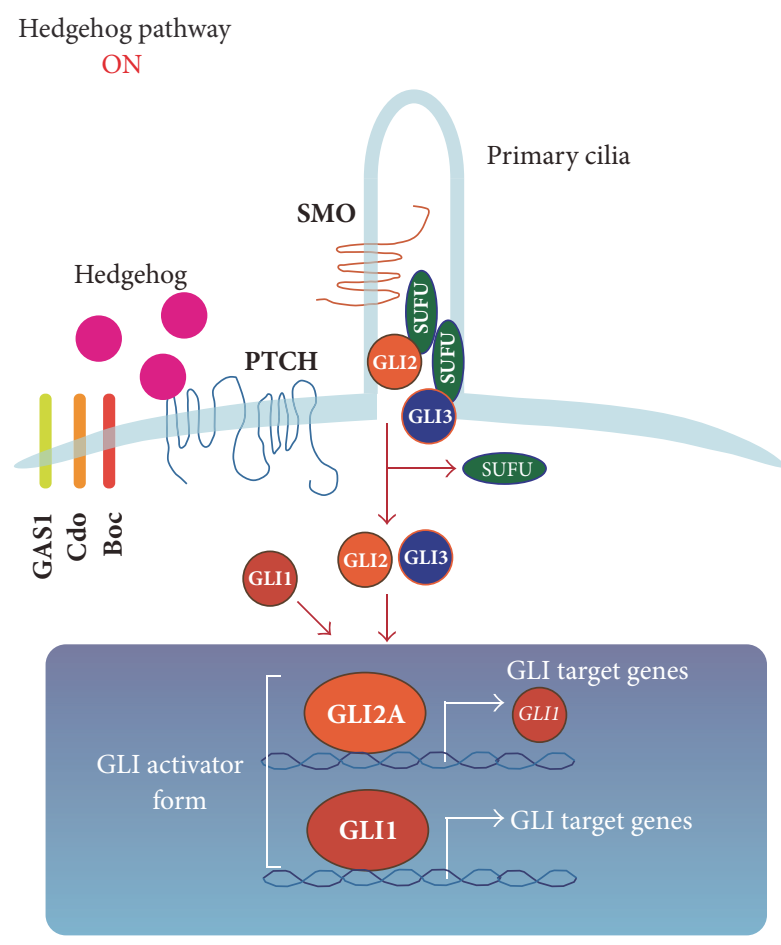

(b)

FIGURE 2: HH signaling pathway during development. (a) In the absence of HH ligands, PTCH blocks ciliary localization of SMO and the GLI repressor form (mainly GLI3 repressor form [GLI3R]) suppresses induction of GLI target gene expression. (b) In the presence of HH ligands, the $\mathrm{HH}$ pathway is activated. Binding of $\mathrm{HH}$ ligand to PTCH prevents PTCH inhibition of SMO, and SMO is free to translocate into primary cilia and is fully activated. SMO then activates the GLI family, mainly GLI2. GLI2 upregulates expression of GLI1 as well as GLI target genes. GLI1 is also activated downstream of SMO. Activated GLI2 (GLI2A) and GLI1 further upregulate expression of various GLI target genes.

from embryonic day (E) 10 to 16.5 is important for branching and growing bronchi [37]. After E16.5, SHH expression is restricted to a subset of the epithelial cells [37]. PTCH expression pattern in growing bronchi mirrors the expression pattern of $\mathrm{SHH}$ [38]. PTCH is also expressed in mesenchyme around E11.5 [39]. Smo is reportedly expressed in epithelium and mesenchyme between E12.5 and E16.5 (pseudoglandular stage) [40]. GLI1, GLI2, and GLI3 are expressed in the mesenchyme during the pseudoglandular stage, and their levels decrease near birth [41]. Although SHH and PTCH expression levels are decreased at birth, they are still observed in epithelial cells [38]. Reduction of the $\mathrm{HH}$ signaling pathway in the postnatal lung induces abnormal lung maturation. Therefore, the $\mathrm{HH}$ signaling pathway is also involved in postnatal lung maturation $[42,43]$. In the healthy adult lung, $\mathrm{HH}$ signaling maintains adult lung quiescence and regulates repair [44]. However, it is still currently unclear how $\mathrm{HH}$ signaling can promote quiescence on the one hand and tumorigenesis on the other.

Constitutive activation of $\mathrm{HH}$ signaling has been observed in many cancers (e.g., skin, lung, stomach, and colon) [45] and promotes cancer cell proliferation, metastasis, and CSC maintenance. Multiple mechanisms of $\mathrm{HH}$ signaling pathway activation in cancer have been proposed. Somatic mutations in $\mathrm{HH}$ pathway components and overproduction of $\mathrm{HH}$ ligands cause aberrant $\mathrm{HH}$ signaling pathway activation. Somatic mutations of PTCH1 and SMO were identified in patients with basal cell carcinoma and medulloblastoma [46-49]. Other mutations in genes encoding $\mathrm{HH}$ pathway components have been reported, including SUFU in medulloblastoma [50] and GLI1 and GLI3 in pancreatic adenocarcinoma [51]. Moreover, GLI1 amplification was observed in glioblastoma [52]. HH ligand overproduction was observed in upper gastrointestinal tract, pancreas, colon, and metastatic prostate cancers, as well as SCLC, glioblastomas, and melanomas [53-58]. Overproduction of $\mathrm{HH}$ ligands constitutively activates the $\mathrm{HH}$ pathway in $\mathrm{HH}$ ligandproducing cancer cells by autocrine signaling $[53,54]$ and in stroma cells such as cancer-associated fibroblasts (CAFs) surrounding $\mathrm{HH}$ ligand-producing cancer cells by paracrine signaling $[16,59]$ (Figure 3 ). In addition, noncanonical $\mathrm{HH}$ signaling has been defined as ligand-dependent activation of SMO but independent of GLI activation [60] or as GLI activation independent of SMO. The noncanonical GLI activation pathway includes transforming growth factor $\beta$ (TGF- $\beta$ ) [61], EGFR [62], Ras-Erk [63, 64], and PI3K-Akt-mechanistic target of rapamycin (mTOR) [65] signaling pathways.

\section{HH Signaling Pathway in SCLC}

Although mutation or amplification of genes involved in the $\mathrm{HH}$ pathway has not been found in SCLC, the $\mathrm{HH}$ signaling 


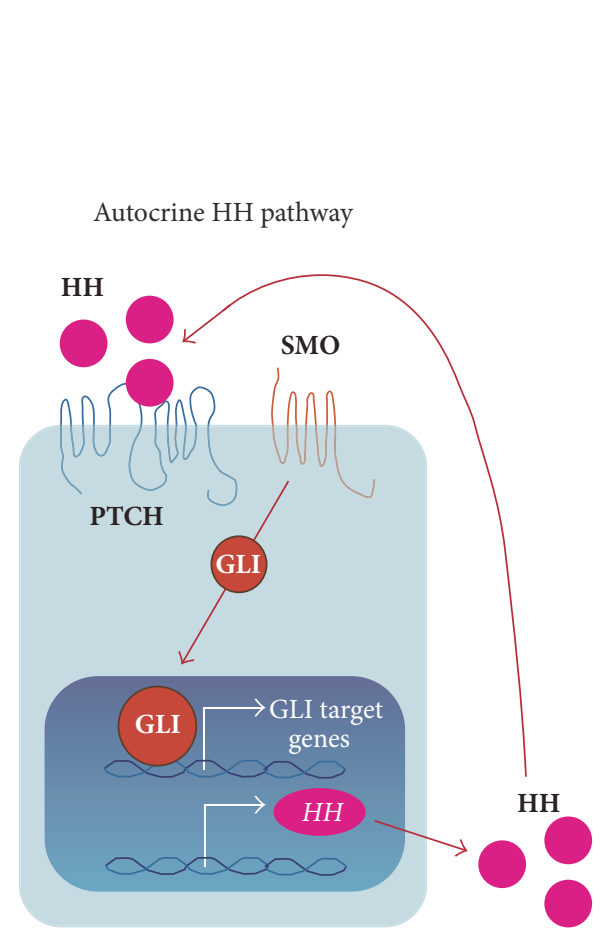

(a)

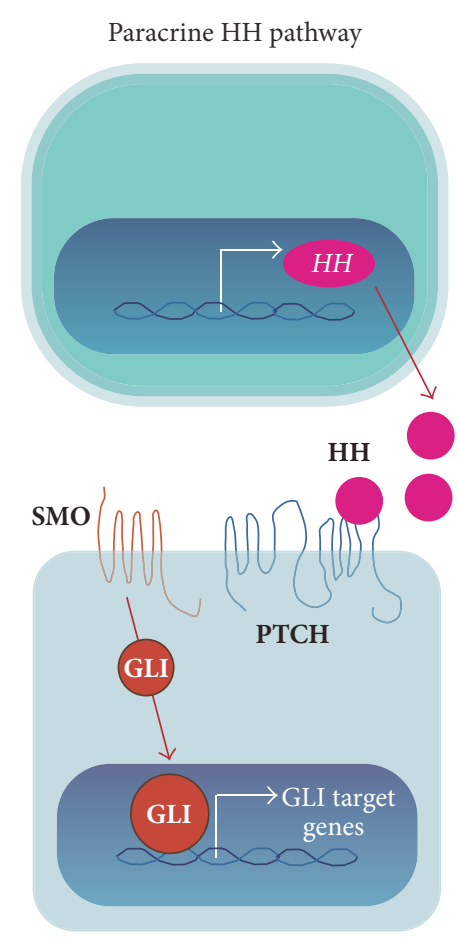

(b)

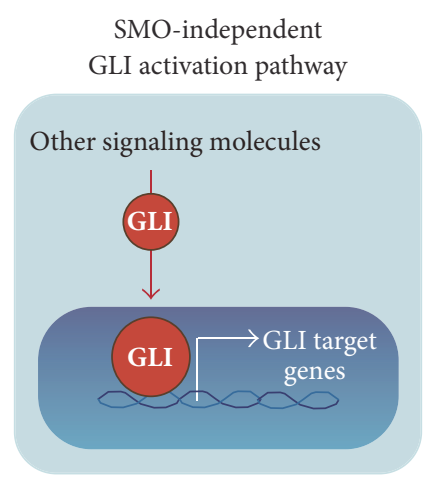

(c)

GLI-independent HH-SMO pathway

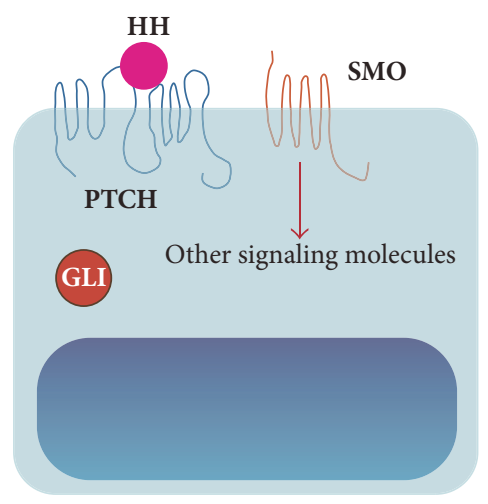

(d)

FIGURE 3: Modes of HH-GLI signaling pathway activation in cancer. (a) HH ligands constitutively activate the HH pathway in HH ligandproducing cancer cells by autocrine signaling. Cancer cells produce $\mathrm{HH}$ ligand, and secreted $\mathrm{HH}$ ligands activate the $\mathrm{HH}$ signaling pathway in these cancer cells. (b) Cancer cell-mediated production of $\mathrm{HH}$ ligands also activates the HH pathway in stroma cells (e.g., CAFs) to maintain cancer cells by paracrine signaling. (c) GLI family transcription factors are activated in a SMO-independent manner, called the noncanonical pathway. (d) SMO can also activate other signaling molecules in cancer cells.

pathway was activated in many SCLC cases [66]. Watkins et al. found $\mathrm{HH}$ pathway activation in neuroendocrine cells in later lung development (E16.5) and the airway epithelium during repair of acute airway injury [53]. Neuroendocrine cells are considered candidates for the origin of SCLC. $\mathrm{HH}$ pathway activation was also observed in SCLC tissue and this observation was confirmed by analysis of SCLC cell lines. Moreover, a SCLC cell xenograft model using nude mice demonstrated that the $\mathrm{HH}$ pathway was activated in SHH-producing SCLC cells but not in surrounding non$\mathrm{SHH}$-producing cancer cells, suggesting that $\mathrm{HH}$ pathway activation was an autocrine and/or juxtacrine loop in SCLC. Analysis using a SCLC model mouse also revealed that $\mathrm{HH}$ pathway activation initiated and progressed mouse SCLC independent of the tumor microenvironment. Furthermore, suppression of SMO in a SCLC mouse model strongly suppressed initiation and progression of SCLC [67]. In addition, immunohistochemistry analysis revealed upregulation of $\mathrm{HH}$ pathway components in SCLC patients, suggesting that the $\mathrm{HH}$ signaling pathway is also activated in SCLC patients [68]. A recent study reported a novel crosstalk between the $\mathrm{HH}$ pathway and bombesin- (BBS-) like neuropeptide-mediated 
signaling in SCLC [69]. SCLC cells secrete BBS, which acts as an autocrine growth factor. Expression of both $\mathrm{SHH}$ and gastrin-releasing peptide receptor (GRPR), a BBS-cognate receptor, was observed in 56\% of SCLC. Analysis of SCLC cell lines revealed that BBS signaling activates GLI1 activity and that BBS-mediated GLI1 activation is suppressed by cyclopamine, a SMO inhibitor. Furthermore, GLI1 activation was mediated by BBS signaling-nuclear factor- $\kappa \mathrm{B}-(\mathrm{NF}-\kappa \mathrm{B}-)$ mediated production of SHH ligand in SCLC cells.

\section{HH Signaling Pathway in NSCLC}

Various studies have also demonstrated that the $\mathrm{HH}$ pathway is activated in NSCLC. The expressions of GLI1 target genes, such as Forkhead Box M1 (FOXM1), B cell-specific Moloney murine leukemia virus integration site 1 (BMI1), and NANOG, were elevated in NSCLC patients [70, 71]. Another study showed that $40 \mathrm{~S}$ ribosomal protein S6 kinase 2 (p70S6K2) regulates GLI1 activity in NSCLC cells. siRNAmediated p70S6K2 knockdown suppressed cell viability and GLI1 activity, and p70S6K2 knockdown promoted GLI1 degradation through inhibition of glycogen synthase kinase $3 \beta$ - (GSK3 $\beta$-) mediated GLI1 phosphorylation. However, a SMO inhibitor, 3-keto-N-aminoethylaminocaproyldihydrocinnamoyl- (KAAD-) cyclopamine [72], did not affect GLI1 activity, and PI3K inhibitor treatment suppressed GLI1 activity [73].

CAFs are widely defined as all fibroblast cells within the tumor stroma and key players in the process of tumorigenesis through modulation of tumor microenvironment, CSC maintenance, and regulation of tumor metabolism [74]. CAF proliferation is maintained by various factors such as growth factors (e.g., TGF- $\beta$ and platelet-derived growth factor [PDGF]) and cytokines (e.g., interleukin 1 [IL-1] and IL-6) [75]. Bermudez et al. showed that NSCLC cells can secrete $\mathrm{SHH}$ ligand, and secreted $\mathrm{SHH}$ ligand activates the $\mathrm{HH}$ signaling pathway in CAFs. This pathway activation induces CAF proliferation [76]. Huang et al. showed that PTCH, SMO, and GLI2 expressions were upregulated in LSCCderived cell lines. However, SMO inhibitor treatment or SMO knockdown demonstrated only a minor inhibitory effect on cell proliferation, whereas GLI2 suppression significantly suppressed cell proliferation and induced extensive apoptosis. Therefore, GLI transcriptional activity would be regulated by a noncanonical (SMO-independent) pathway [77]. These reports suggest that the $\mathrm{HH}$ pathway is activated by the paracrine mechanism and GLI activation in NSCLC cells is regulated by the noncanonical (SMO-independent) pathway.

On the other hand, several studies have reported that $\mathrm{HH}$ signaling is activated by the autocrine pathway in NSCLC cells. The aggressiveness of NSCLC has been shown to be associated with the acquisition of epithelial-to-mesenchymal transition (EMT) [78]. A549 lung adenocarcinoma cells that obtain mesenchymal phenotype (A549-M cells) show upregulated SHH ligand and GLI1 expression compared with A549 cells. In A549-M cells, the $\mathrm{HH}$ pathway was activated by autocrine signaling, and suppression of the $\mathrm{HH}$ pathway contributed to suppression of TGF- $\beta$ signaling-induced cancer cell migration and metastatic characteristics [79].
CAFs can secrete various growth factors and cytokines. Secreted proteins induce extracellular matrix (ECM) remodeling. Furthermore, CAFs interacts with cancer cells and CAF-secreted proteins activate various signaling pathway by paracrine signaling. ECM remodeling and CAFs-mediated paracrine signaling pathway activation could induce metastatic properties of cancer cells [75]. Choe et al. [80] showed that EMT-related gene expression and the $\mathrm{HH}$ signaling pathway was upregulated in adenocarcinoma cells by means of direct coculture of NSCLC cells and lung CAFs. The authors proposed that metastatic properties might be acquired by direct interaction of adenocarcinoma cells and CAFs and CAF-mediated paracrine $\mathrm{HH}$ signaling pathway activation in adenocarcinoma cells.

CSCs exhibit a self-renewing capacity and are responsible for tumor maintenance and relapse [81]. CSC maintenance in adenocarcinoma and LSCC are regulated by the autocrine $\mathrm{HH}$ signaling pathway. Several molecules and enzymatic activities such as CD44, CD133, and high aldehyde dehydrogenase (ALDH) activity have been identified as CSC markers of NSCLC [82-85]. The HH signaling pathway was activated in $\mathrm{CD} 44^{\text {high }} / \mathrm{ALDH}^{\text {high }}$ cancer cells harboring CSC properties [86]. Furthermore, $\mathrm{CD}_{133^{+}} \mathrm{NSCLC}$ cells also exhibit CSC properties and secrete $\mathrm{SHH}$ ligand, and $\mathrm{HH}$ pathway inhibition in $\mathrm{CD}_{133^{+}}$cells attenuated sphere formation, suggesting that the autocrine $\mathrm{HH}$ pathway is involved in $\mathrm{CD}_{133^{+}}$ CSC maintenance [87]. Although $\mathrm{CD} 133^{+}$SCLC cells are identified as a CSC phenotype [88], there is no evidence that the $\mathrm{HH}$ pathway is involved in SCLC stem cell maintenance. Moreover, a previous report showed that GLI1 upregulated expression of the embryonic stem cell transcription factor SRY- (sex determining region Y-) box 2 (SOX2) by cooperation with EGF signaling in lung adenocarcinoma-derived cell lines [89]. As described above, the interaction of CAFs and NSCLC cells induces metastatic properties of NSCLC cells via CAF-mediated $\mathrm{HH}$ signaling pathway activation in NSCLC cells. Chen et al. showed that an interaction of CAFs and NSCLC cells and CAF-mediated $\mathrm{HH}$ signaling pathway activation in NSCLC cells are also involved in CSC maintenance [90]. We independently observed that GLI1 inhibition but not SMO inhibition attenuated sphere formation, suggesting that GLI1 activity was regulated by other signaling pathways for NSCLC stem cell maintenance (unpublished data).

SOX2 expression is upregulated in LSCC [91], and therefore SOX2 is used as one of the tumor markers for LSCC. Although SOX2 has critical roles in CSC maintenance, the precise mechanism of SOX2-mediated CSC maintenance is largely unknown. Justilien et al. reported that the SOX2-HH pathway has important roles for CSC maintenance in LSCC. Protein kinase C iota (PRKCI) phosphorylated Ser394 in SOX2, resulting in upregulated expression of hedgehog acyltransferase (HHAT). The SHH ligand is changed to its active form by HHAT, resulting in $\mathrm{HH}$ signaling pathway activation. The PRKCI-SOX2-HH signaling pathway plays important roles in CSC maintenance [92]. 
TABLE 1: The HH signaling pathway inhibitors.

\begin{tabular}{lcc}
\hline Inhibitor Name & Organization & Clinical Trial \\
\hline (1) SMO inhibitors & - & No \\
Cyclopamine, KAAD-cyclopamine & Roche/Genentech/Curis & Yes (phases 0, I, and II) \\
GDC-0449 (Vismodegib/Erivedge) & Novartis & Yes (phases 0, I, and II) \\
LDE225 (Erismodegib/Sonidegib/Odomzo) & Bristol Myers Squibb/Exelixis & Yes (phases I and II) \\
BMS-833923/XL139 & Pfizer & Yes (Phase II) \\
PF-04449913 (Glasdegib) & Pfizer & No \\
PF-527857 & Ignyta & Yes (phases I and II) \\
LY2940680 (Taladegib) & Infinity & Yes (phase I) \\
IPI-926 (Sadegib) & - & No \\
TAK-441 & - & No \\
MRT-92 & - & No \\
(2) GLI inhibitors & - & Yes (phases I, II, III, and IV) \\
GANT-58, GANT-61 & - & No \\
Arsenic trioxide (ATO) & - & No \\
HPI-1 & Glabrescione B (GlaB) & - \\
\hline
\end{tabular}

See [14] for description of the clinical trials of $\mathrm{HH}$ signaling pathway inhibitors.

As described above, SMO inhibitor treatment suppressed EMT properties through remodeling of the actin cytoskeleton and motility of NSCLC cells [79]. Although SMO inhibition downregulated EMT-associated gene expression, expressions of GLI1 target genes were not affected [93]. These results suggest that SMO might activate other signaling molecules as well as GLI transcription factors in NSCLC cells harboring mesenchymal properties.

Many studies on the roles of the $\mathrm{HH}$ signaling pathway in NSCLC suggested that GLI1 and GLI2 play central roles in tumor progression, tumor metastasis, and CSC maintenance. The mechanisms of GLI activation are diverse in cancer cell types and the tumor microenvironment surrounding cancer cells, since GLI is activated by various pathways including the autocrine and paracrine $\mathrm{HH}$ pathways as well as canonical and noncanonical GLI activation pathway.

\section{HH Signaling Pathway-Targeted Cancer Therapy in Lung Cancer}

Previous studies have revealed that subsets of lung cancer patients harbor mutations in the key oncogenic drivers upon which tumor survival and progression are dependent. These include mutations in EGFR and the EML4-ALK fusion protein [3]. Therefore, various TKIs targeting EGFR and EML4ALK have been developed. However, the clinical efficacy of TKIs differs among patients, and acquired resistance for chronic treatment often develops in most patients who are treated with TKIs [5, 94, 95]. Furthermore, there are no effective anticancer drugs for SCLC, LSCC, and large cell carcinoma.

Previous studies reported that tumor volume and tumor recurrence were suppressed by $\mathrm{HH}$ pathway inhibitor treatment or combination treatment of $\mathrm{HH}$ pathway inhibitors and other types of chemotherapeutic agents such as TKIs and platinum-containing drugs. Park et al. [67] demonstrated that combination treatment of etoposide and a SMO inhibitor (LDE225: Sonidegib) [96] attenuated tumor recurrence of SCLC using a mouse xenograft model. Moreover, LDE225 treatment attenuated the TKI-resistant NSCLC cell line HCC827-GR (gefitinib resistant) derived tumor growth. In addition, cotreatment of SMO inhibitor and MET inhibitor to HCC827-GR xenografted tumors further suppressed tumor volume, since constitutive MET activation was observed in HCC827-GR cells [97]. Moreover, RNAi-mediated GLI1 knockdown suppressed tumor formation and tumor sphere formation. Several SMO inhibitors and GLI inhibitors have been developed [14]. GDC-0449 (Vismodegib) [98] is approved for basal cell carcinoma therapy, and several SMO inhibitors including GDC-0449 are used in clinical investigations for SCLC. GLI inhibitors such as GLI-antagonist(GANT-) 58, GANT-61, HH pathway inhibitor- (HPI-) 1, Genistein, and Glabrescione B (GlaB) have also been developed [43, 99-101]. In addition, arsenic trioxide (ATO), which suppresses GLI1 transcriptional activity [102, 103], is used in clinical investigations as a GLI inhibitor (Table 1) [14]. However, other GLI inhibitors have not yet progressed to clinical trials. Since the HH pathway and GLI activity have important roles in lung cancer formation and lung CSC maintenance, these chemical compounds may be useful for lung cancer therapy.

\section{Conclusion}

We have discussed the relationship between the $\mathrm{HH}$ signaling pathway and lung cancer and the mechanism of $\mathrm{HH}$ signaling pathway activation in lung cancer. As summarized in Figure 4, the GLI activation machinery and the role of the $\mathrm{HH}$ pathway in lung cancer are different in NSCLC and SCLC 


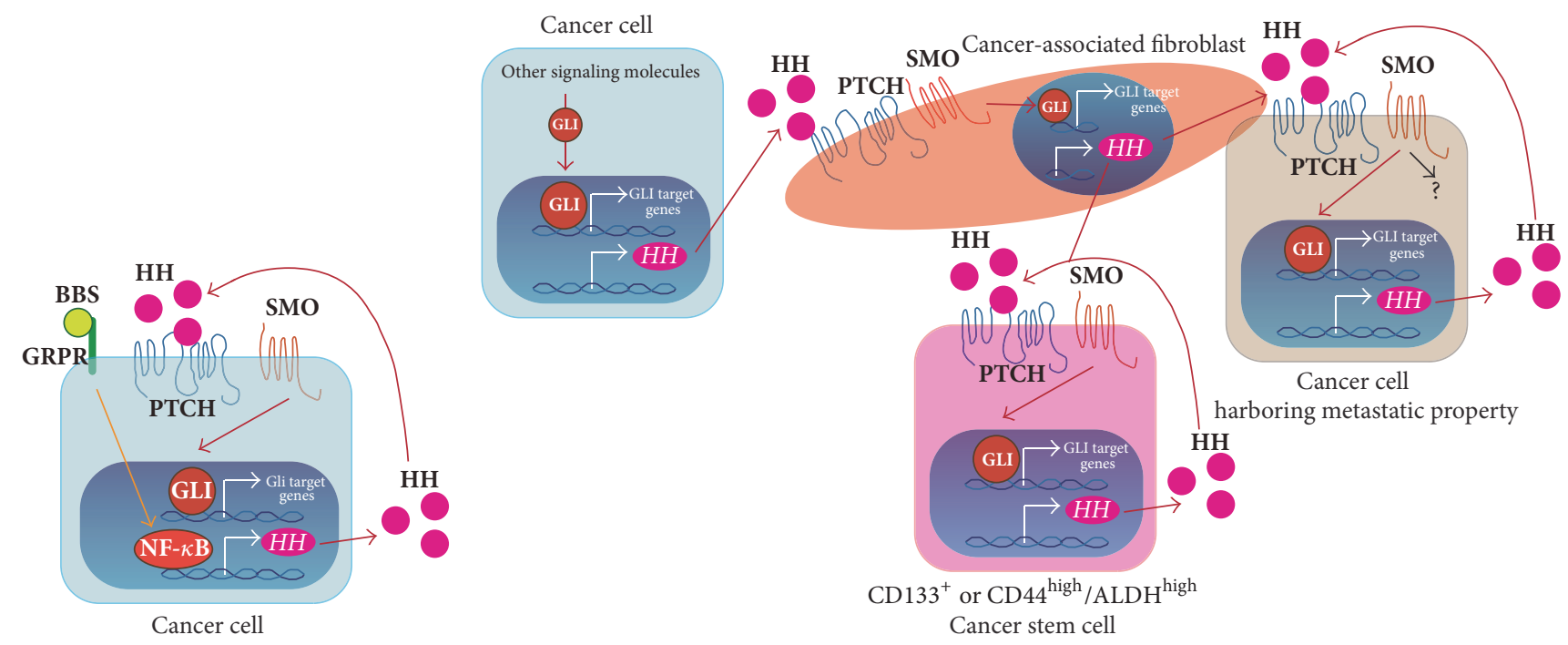

(a)

(b)

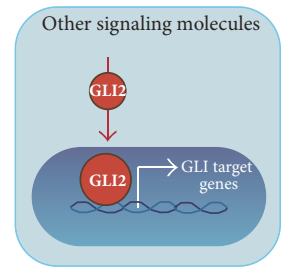

Cancer cell

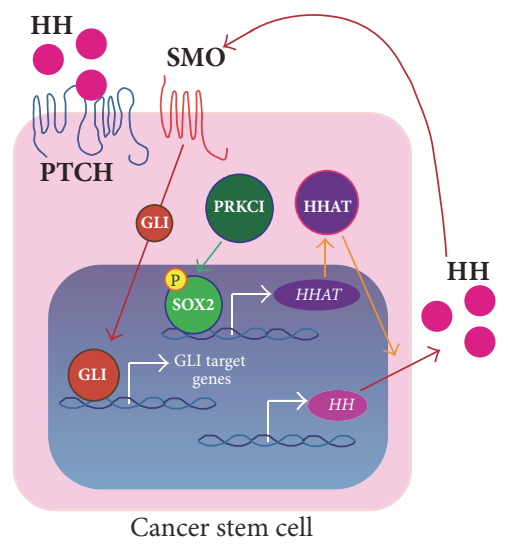

(c)

FIGURE 4: The role of the HH signaling pathway in lung cancer. (a) The HH signaling pathway in SCLC. The autocrine HH signaling pathway promotes cancer cell proliferation. (b) The HH signaling pathway in adenocarcinoma. The noncanonical GLI activation pathway would maintain cancer cell proliferation. CAF maintenance would be regulated by paracrine $\mathrm{HH}$ signaling pathway activation. CAF-secreted $\mathrm{HH}$ ligands would activate the HH signaling pathway in cancer cells and CSCs. CAFs-mediated paracrine HH pathway activation in cancer cells has important roles in acquisition of metastatic properties. Moreover, CAF-mediated HH signaling pathway activation might be involved in CSC maintenance. Cancer cells harboring metastatic properties and CSCs would be also maintained by autocrine $\mathrm{HH}$ signaling pathway activation. In addition, SMO might activate other signaling molecules in cancer cells harboring metastatic properties. (c) The HH signaling pathway in LSCC. Cancer cells would be maintained by the noncanonical GLI2 activation pathway. PRKCI-SOX2-HH signaling pathway has important roles in CSC maintenance.

as well as among the types of NSCLC. Furthermore, the $\mathrm{HH}$ signaling pathway is involved in the interaction of cancer cells and CAFs for tumor maintenance. Various SMO inhibitors are used in clinical investigations for lung cancer. Results from in vitro and in vivo experiments have demonstrated that SMO inhibitor treatment is effective for lung tumor suppression. In fact, SMO inhibitors are used in clinical trials for SCLC. The HH signaling pathway is involved in CSC maintenance, tumor progression, and metastasis in NSCLC. Therefore, SMO inhibitors may be a better option for lung cancer therapy in the future. However, previous studies suggest that
GLI transcription factors are activated by various mechanisms, including the SMO-independent pathway. In particular, dysregulated SMO-independent GLI activation pathway may cause SMO inhibitor resistance. Several GLI inhibitors have also been recently developed. Therefore, a HH-pathwayactivated lung cancer therapy using GLI inhibitors would be an effective option. To develop the most effective $\mathrm{HH}$ pathway inhibitor for treatment of lung cancer, the current challenge is not only to accelerate $\mathrm{HH}$ inhibitor development but also to more deeply understand the regulatory mechanism of GLImediated transcription. 


\section{Competing Interests}

The authors have no competing interests to review.

\section{Authors' Contributions}

Yoshinori Abe and Nobuyuki Tanaka contributed equally to this work.

\section{References}

[1] R. Siegel, D. Naishadham, and A. Jemal, "Cancer statistics, 2013," CA Cancer Journal for Clinicians, vol. 63, no. 1, pp. 11-30, 2013.

[2] M. R. Davidson, A. F. Gazdar, and B. E. Clarke, "The pivotal role of pathology in the management of lung cancer," Journal of Thoracic Disease, vol. 5, no. 5, pp. S463-S478, 2013.

[3] C. J. Langer, B. Besse, A. Gualberto, E. Brambilla, and J.-C. Soria, "The evolving role of histology in the management of advanced non-small-cell lung cancer," Journal of Clinical Oncology, vol. 28, no. 36, pp. 5311-5320, 2010.

[4] M. Soda, Y. L. Choi, M. Enomoto et al., "Identification of the transforming EML4-ALK fusion gene in non-small-cell lung cancer," Nature, vol. 448, no. 7153, pp. 561-566, 2007.

[5] J. A. Engelman, K. Zejnullahu, T. Mitsudomi et al., "MET amplification leads to gefitinib resistance in lung cancer by activating ERBB3 signaling," Science, vol. 316, no. 5827, pp. 1039-1043, 2007.

[6] K. Rikova, A. Guo, Q. Zeng et al., "Global survey of phosphotyrosine signaling identifies oncogenic kinases in lung cancer," Cell, vol. 131, no. 6, pp. 1190-1203, 2007.

[7] P. Stephens, C. Hunter, G. Bignell et al., "Lung cancer: intragenic ERBB2 kinase mutations in tumours," Nature, vol. 431, no. 7008, pp. 525-526, 2004.

[8] A. Vaishnavi, M. Capelletti, A. T. Le et al., "Oncogenic and drug-sensitive NTRK1 rearrangements in lung cancer," Nature Medicine, vol. 19, no. 11, pp. 1469-1472, 2013.

[9] T. C. G. A. R. Network, "Comprehensive genomic characterization of squamous cell lung cancers," Nature, vol. 489, no. 7417, pp. 519-525, 2012.

[10] B. Hallberg and R. H. Palmer, "Mechanistic insight into ALK receptor tyrosine kinase in human cancer biology," Nature Reviews Cancer, vol. 13, no. 10, pp. 685-700, 2013.

[11] W. Pao and J. Chmielecki, "Rational, biologically based treatment of EGFR-mutant non-small-cell lung cancer," Nature Reviews Cancer, vol. 10, no. 11, pp. 760-774, 2010.

[12] J. N. Spaans and G. D. Goss, "Epidermal growth factor receptor tyrosine kinase inhibitors in early-stage nonsmall cell lung cancer," Current Opinion in Oncology, vol. 27, no. 2, pp. 102-107, 2015.

[13] L. Strizzi, K. M. Hardy, E. A. Seftor et al., "Development and cancer: at the crossroads of Nodal and Notch signaling," Cancer Research, vol. 69, no. 18, pp. 7131-7134, 2009.

[14] T. K. Rimkus, R. L. Carpenter, S. Qasem, M. Chan, and H.-W. Lo, "Targeting the sonic hedgehog signaling pathway: review of smoothened and GLI inhibitors," Cancers, vol. 8, no. 2, article 22, 2016.

[15] A. A. Merchant and W. Matsui, "Targeting Hedgehog-a cancer stem cell pathway," Clinical Cancer Research, vol. 16, no. 12, pp. 3130-3140, 2010.
[16] R. L. Yauch, S. E. Gould, S. J. Scales et al., "A paracrine requirement for hedgehog signalling in cancer," Nature, vol. 455, no. 7211, pp. 406-410, 2008.

[17] P. W. Ingham and A. P. McMahon, "Hedgehog signaling in animal development: paradigms and principles," Genes \& Development, vol. 15, no. 23, pp. 3059-3087, 2001.

[18] P. A. Beachy, S. S. Karhadkar, and D. M. Berman, “Tissue repair and stem cell renewal in carcinogenesis," Nature, vol. 432, no. 7015, pp. 324-331, 2004.

[19] J. M. Y. Ng and T. Curran, “The Hedgehog's tale: developing strategies for targeting cancer," Nature Reviews Cancer, vol. 11, no. 7, pp. 493-501, 2011.

[20] J. Brechbiel, K. Miller-Moslin, and A. A. Adjei, "Crosstalk between hedgehog and other signaling pathways as a basis for combination therapies in cancer," Cancer Treatment Reviews, vol. 40, no. 6, pp. 750-759, 2014.

[21] F. Aberger and A. Ruiz i Altaba, "Context-dependent signal integration by the GLI code: the oncogenic load, pathways, modifiers and implications for cancer therapy," Seminars in Cell \& Developmental Biology, vol. 33, pp. 93-104, 2014.

[22] R. Rohatgi, L. Milenkovic, and M. P. Scott, "Patched1 regulates hedgehog signaling at the primary cilium," Science, vol. 317, no. 5836, pp. 372-376, 2007.

[23] B. L. Allen, T. Tenzen, and A. P. McMahon, “The Hedgehogbinding proteins Gasl and Cdo cooperate to positively regulate Shh signaling during mouse development," Genes and Development, vol. 21, no. 10, pp. 1244-1257, 2007.

[24] A. Okada, F. Charron, S. Morin et al., "Boc is a receptor for sonic hedgehog in the guidance of commissural axons," Nature, vol. 444, no. 7117, pp. 369-373, 2006.

[25] K. C. Corbit, P. Aanstad, V. Singla, A. R. Norman, D. Y. R. Stainier, and J. F. Reiter, "Vertebrate Smoothened functions at the primary cilium," Nature, vol. 437, no. 7061, pp. 1018-1021, 2005.

[26] C. J. Haycraft, B. Banizs, Y. Aydin-Son, Q. Zhang, E. J. Michaud, and B. K. Yoder, "Gli2 and Gli3 localize to cilia and require the intraflagellar transport protein polaris for processing and function," PLoS Genetics, vol. 1, no. 4, article no. e53, 2005.

[27] Y. Lee, R. Kawagoe, K. Sasai et al., "Loss of suppressor-of-fused function promotes tumorigenesis," Oncogene, vol. 26, no. 44, pp. 6442-6447, 2007.

[28] H. Sasaki, Y. Nishizaki, C.-C. Hui, M. Nakafuku, and H. Kondoh, "Regulation of Gli2 and Gli3 activities by an amino-terminal repression domain: implication of Gli2 and Gli3 as primary mediators of Shh signaling," Development, vol. 126, no. 17, pp. 3915-3924, 1999.

[29] C. B. Bai, W. Auerbach, J. S. Lee, D. Stephen, and A. L. Joyner, "Gli2, but not Gli1, is required for initial Shh signaling and ectopic activation of the Shh pathway," Development, vol. 129, no. 20, pp. 4753-4761, 2002.

[30] H. Masuya, T. Sagai, K. Moriwaki, and T. Shiroishi, "Multigenic control of the localization of the zone of polarizing activity in limb morphogenesis in the mouse," Developmental Biology, vol. 182, no. 1, pp. 42-51, 1997.

[31] D. Büscher, B. Bosse, J. Heymer, and U. Rüther, "Evidence for genetic control of Sonic hedgehog by Gli3 in mouse limb development," Mechanisms of Development, vol. 62, no. 2, pp. 175182, 1997.

[32] Q. Ding, J. Motoyama, S. Gasca et al., "Diminished Sonic hedgehog signaling and lack of floor plate differentiation in Gli2 mutant mice," Development, vol. 125, no. 14, pp. 2533-2543, 1998. 
[33] H. L. Park, C. Bai, K. A. Platt et al., "Mouse Glil mutants are viable but have defects in SHH signaling in combination with a Gli2 mutation," Development, vol. 127, no. 8, pp. 1593-1605, 2000.

[34] C. B. Bai and A. L. Joyner, "Glil can rescue the in vivo function of Gli2,” Development, vol. 128, no. 24, pp. 5161-5172, 2001.

[35] H. Tukachinsky, L. V. Lopez, and A. Salic, "A mechanism for vertebrate Hedgehog signaling: recruitment to cilia and dissociation of SuFu-Gli protein complexes," Journal of Cell Biology, vol. 191, no. 2, pp. 415-428, 2010.

[36] M. C. Kugler, A. L. Joyner, C. A. Loomis, and J. S. Munger, "Sonic hedgehog signaling in the lung: from development to disease," American Journal of Respiratory Cell and Molecular Biology, vol. 52, no. 1, pp. 1-13, 2015.

[37] L.-A. D. Miller, S. E. Wert, and J. A. Whitsett, "Immunolocalization of sonic hedgehog (Shh) in developing mouse lung," Journal of Histochemistry and Cytochemistry, vol. 49, no. 12, pp. 1593-1603, 2001.

[38] S. Bellusci, Y. Furuta, M. G. Rush, R. Henderson, G. Winnier, and B. L. M. Hogan, "Involvement of Sonic hedgehog (Shh) in mouse embryonic lung growth and morphogenesis," Development, vol. 124, no. 1, pp. 53-63, 1997.

[39] M. Weaver, L. Batts, and B. L. M. Hogan, "Tissue interactions pattern the mesenchyme of the embryonic mouse lung," Developmental Biology, vol. 258, no. 1, pp. 169-184, 2003.

[40] M. Zhang, H. Wang, H. Teng, J. Shi, and Y. Zhang, "Expression of SHH signaling pathway components in the developing human lung," Histochemistry and Cell Biology, vol. 134, no. 4, pp. 327-335, 2010.

[41] J. C. Grindley, S. Bellusci, D. Perkins, and B. L. M. Hogan, "Evidence for the involvement of the Gli gene family in embryonic mouse lung development," Developmental Biology, vol. 188, no. 2, pp. 337-348, 1997.

[42] L. Liu, M. C. Kugler, C. A. Loomis et al., "Hedgehog signaling in neonatal and adult lung," American Journal of Respiratory Cell and Molecular Biology, vol. 48, no. 6, pp. 703-710, 2013.

[43] J. M. Hyman, A. J. Firestone, V. M. Heine et al., "Small-molecule inhibitors reveal multiple strategies for Hedgehog pathway blockade," Proceedings of the National Academy of Sciences of the United States of America, vol. 106, no. 33, pp. 14132-14137, 2009.

[44] T. Peng, D. B. Frank, R. S. Kadzik et al., "Hedgehog actively maintains adult lung quiescence and regulates repair and regeneration," Nature, vol. 526, no. 7574, pp. 578-582, 2015.

[45] L. L. Rubin and F. J. de Sauvage, "Targeting the Hedgehog pathway in cancer," Nature Reviews Drug Discovery, vol. 5, no. 12, pp. 1026-1033, 2006.

[46] C. Raffel, R. B. Jenkins, L. Frederick et al., "Sporadic medulloblastomas contain PTCH mutations," Cancer Research, vol. 57, no. 5, pp. 842-845, 1997.

[47] M. Wolter, J. Reifenberger, C. Sommer, T. Ruzicka, and G. Reifenberger, "Mutations in the human homologue of the Drosophila segment polarity gene patched $(\mathrm{PTCH})$ in sporadic basal cell carcinomas of the skin and primitive neuroectodermal tumors of the central nervous system," Cancer Research, vol. 57, no. 13, pp. 2581-2585, 1997.

[48] J. Reifenberger, M. Wolter, R. G. Weber et al., "Missense mutations in SMOH in sporadic basal cell carcinomas of the skin and primitive neuroectodermal tumors of the central nervous system," Cancer Research, vol. 58, no. 9, pp. 1798-1803, 1998.

[49] J. Xie, M. Murone, S.-M. Luoh et al., "Activating Smoothened mutations in sporadic basal-cell carcinoma," Nature, vol. 391, no. 6662 , pp. $90-92,1998$.
[50] M. D. Taylor, L. Liu, C. Raffel et al., "Mutations in SUFU predispose to medulloblastoma," Nature Genetics, vol. 31, no. 3, pp. 306-310, 2002.

[51] S. Jones, X. Zhang, D. W. Parsons et al., "Core signaling pathways in human pancreatic cancers revealed by global genomic analyses," Science, vol. 321, no. 5897, pp. 1801-1806, 2008.

[52] A. J. Wong, S. H. Bigner, D. D. Bigner, K. W. Kinzler, S. R. Hamilton, and B. Vogelstein, "Increased expression of the epidermal growth factor receptor gene in malignant gliomas is invariably associated with gene amplification," Proceedings of the National Academy of Sciences of the United States of America, vol. 84, no. 19, pp. 6899-6903, 1987.

[53] D. N. Watkins, D. M. Berman, S. G. Burkholder, B. Wang, P. A. Beachy, and S. B. Baylin, "Hedgehog signalling within airway epithelial progenitors and in small-cell lung cancer," Nature, vol. 422, no. 6929, pp. 313-317, 2003.

[54] D. M. Berman, S. S. Karhadkar, A. Maitra et al., "Widespread requirement for Hedgehog ligand stimulation in growth of digestive tract tumours," Nature, vol. 425, no. 6960, pp. 846-851, 2003.

[55] F. Varnat, A. Duquet, M. Malerba et al., "Human colon cancer epithelial cells harbour active HEDGEHOG-GLI signalling that is essential for tumour growth, recurrence, metastasis and stem cell survival and expansion," EMBO Molecular Medicine, vol. 1, no. 6-7, pp. 338-351, 2009.

[56] S. S. Karhadkar, G. S. Bova, N. Abdallah et al., "Hedgehog signalling in prostate regeneration, neoplasia and metastasis," Nature, vol. 431, no. 7009, pp. 707-712, 2004.

[57] E. E. Bar, A. Chaudhry, A. Lin et al., "Cyclopamine-mediated Hedgehog pathway inhibition depletes stem-like cancer cells in glioblastoma," STEM CELLS, vol. 25, no. 10, pp. 2524-2533, 2007.

[58] B. Stecca, C. Mas, V. Clement et al., "Melanomas require HEDGEHOG-GLI signaling regulated by interactions between GLI1 and the RAS-MEK/AKT pathways," Proceedings of the National Academy of Sciences of the United States of America, vol. 104, no. 14, pp. 5895-5900, 2007.

[59] J.-W. Theunissen and F. J. De Sauvage, "Paracrine hedgehog signaling in cancer," Cancer Research, vol. 69, no. 15, pp. 60076010, 2009.

[60] D. Jenkins, "Hedgehog signalling: emerging evidence for noncanonical pathways," Cellular Signalling, vol. 21, no. 7, pp. 1023$1034,2009$.

[61] D. Javelaud, M.-J. Pierrat, and A. Mauviel, "Crosstalk between TGF- $\beta$ and hedgehog signaling in cancer," FEBS Letters, vol. 586, no. 14, pp. 2016-2025, 2012.

[62] M. Eberl, S. Klingler, D. Mangelberger et al., "Hedgehog-EGFR cooperation response genes determine the oncogenic phenotype of basal cell carcinoma and tumour-initiating pancreatic cancer cells," EMBO Molecular Medicine, vol. 4, no. 3, pp. 218233, 2012.

[63] O. Nolan-Stevaux, J. Lau, M. L. Truitt et al., "GLI1 is regulated through Smoothened-independent mechanisms in neoplastic pancreatic ducts and mediates PDAC cell survival and transformation," Genes \& Development, vol. 23, no. 1, pp. 24-36, 2009.

[64] T. Mazumdar, J. DeVecchio, A. Agyeman, T. Shi, and J. A. Houghton, "The GLI genes as the molecular switch in disrupting Hedgehog signaling in colon cancer," Oncotarget, vol. 2, no. 8, pp. 638-645, 2011.

[65] Y. Wang, Q. Ding, C.-J. Yen et al., "The crosstalk of mTOR/S6K1 and Hedgehog pathways," Cancer Cell, vol. 21, no. 3, pp. 374-387, 2012. 
[66] J. Voortman, J.-H. Lee, J. K. Killian et al., "Array comparative genomic hybridization-based characterization of genetic alterations in pulmonary neuroendocrine tumors," Proceedings of the National Academy of Sciences of the United States of America, vol. 107, no. 29, pp. 13040-13045, 2010.

[67] K.-S. Park, L. G. Martelotto, M. Peifer et al., "A crucial requirement for Hedgehog signaling in small cell lung cancer," Nature Medicine, vol. 17, no. 11, pp. 1504-1508, 2011.

[68] J. Vestergaard, M. W. Pedersen, N. Pedersen et al., "Hedgehog signaling in small-cell lung cancer: frequent in vivo but a rare event in vitro," Lung Cancer, vol. 52, no. 3, pp. 281-290, 2006.

[69] M. D. Castellone, M. O. Laukkanen, H. Teramoto et al., "Cross talk between the bombesin neuropeptide receptor and Sonic hedgehog pathways in small cell lung carcinoma," Oncogene, vol. 34, no. 13, pp. 1679-1687, 2015.

[70] I. P. Gialmanidis, V. Bravou, I. Petrou et al., "Expression of bmil, FoxF1, nanog, and $\gamma$-catenin in relation to hedgehog signaling pathway in human non-small-cell lung cancer," Lung, vol. 191, no. 5, pp. 511-521, 2013.

[71] I. P. Gialmanidis, V. Bravou, S. G. Amanetopoulou, J. Varakis, H. Kourea, and H. Papadaki, "Overexpression of hedgehog pathway molecules and FOXM1 in non-small cell lung carcinomas," Lung Cancer, vol. 66, no. 1, pp. 64-74, 2009.

[72] J. Talpale, J. K. Chen, M. K. Cooper et al., "Effects of oncogenic mutations in Smoothened and Patched can be reversed by cyclopamine," Nature, vol. 406, no. 6799, pp. 1005-1009, 2000.

[73] S. Mizuarai, A. Kawagishi, and H. Kotani, "Inhibition of p70S6K2 down-regulates Hedgehog/GLI pathway in non-small cell lung cancer cell lines," Molecular Cancer, vol. 8, article no. 44, 2009.

[74] D. Hanahan and R. A. Weinberg, "Hallmarks of cancer: the next generation," Cell, vol. 144, no. 5, pp. 646-674, 2011.

[75] D. Öhlund, E. Elyada, and D. Tuveson, "Fibroblast heterogeneity in the cancer wound," Journal of Experimental Medicine, vol. 211, no. 8, pp. 1503-1523, 2014.

[76] O. Bermudez, E. Hennen, I. Koch, M. Lindner, and O. Eickelberg, "Glil mediates lung cancer cell proliferation and sonic hedgehog-dependent mesenchymal cell activation," PLoS ONE, vol. 8, no. 5, Article ID e63226, 2013.

[77] L. Huang, V. Walter, D. N. Hayes, and M. Onaitis, "HedgehogGLI signaling inhibition suppresses tumor growth in squamous lung cancer," Clinical Cancer Research, vol. 20, no. 6, pp. 15661575, 2014.

[78] S. Thomson, E. Buck, F. Petti et al., "Epithelial to mesenchymal transition is a determinant of sensitivity of non-small-cell lung carcinoma cell lines and xenografts to epidermal growth factor receptor inhibition," Cancer Research, vol. 65, no. 20, pp. 9455$9462,2005$.

[79] M. Y. Maitah, S. Ali, A. Ahmad, S. Gadgeel, and F. H. Sarkar, "Up-regulation of sonic hedgehog contributes to TGF- $\beta 1$ induced epithelial to mesenchymal transition in NSCLC cells," PLoS ONE, vol. 6, no. 1, Article ID e16068, 2011.

[80] C. Choe, Y.-S. Shin, S.-H. Kim et al., “Tumor-stromal interactions with direct cell contacts enhance motility of non-small cell lung cancer cells through the hedgehog signaling pathway," Anticancer Research, vol. 33, no. 9, pp. 3715-3724, 2013.

[81] P. Valent, D. Bonnet, R. De Maria et al., "Cancer stem cell definitions and terminology: the devil is in the details," Nature Reviews Cancer, vol. 12, no. 11, pp. 767-775, 2012.

[82] Y.-C. Chen, H.-S. Hsu, Y.-W. Chen et al., "Oct-4 expression maintained cancer stem-like properties in lung cancer-derived
CD133-positive cells," PLoS ONE, vol. 3, no. 7, Article ID e2637, 2008.

[83] V. Tirino, R. Camerlingo, R. Franco et al., "The role of CD133 in the identification and characterisation of tumour-initiating cells in non-small-cell lung cancer," European Journal of Cardiothoracic Surgery, vol. 36, no. 3, pp. 446-453, 2009.

[84] E. L.-H. Leung, R. R. Fiscus, J. W. Tung et al., "Non-small cell lung cancer cells expressing CD44 are enriched for stem celllike properties," PLoS ONE, vol. 5, no. 11, Article ID e14062, 2010.

[85] M. Alamgeer, C. D. Peacock, W. Matsui, V. Ganju, and D. N. Watkins, "Cancer stem cells in lung cancer: evidence and controversies," Respirology, vol. 18, no. 5, pp. 757-764, 2013.

[86] J. Liu, Z. Xiao, S. K.-M. Wong et al., "Lung cancer tumorigenicity and drug resistance are maintained through ALDH(hi)CD44(hi) tumor initiating cells," Oncotarget, vol. 4, no. 10, pp. 1698-1711, 2013.

[87] S. O. Lee, X. Yang, S. Duan et al., "IL-6 promotes growth and epithelial-mesenchymal transition of CD133+ cells of non-small cell lung cancer," Oncotarget, vol. 7, no. 6, pp. 6626-6638, 2015.

[88] S. Sarvi, A. C. Mackinnon, N. Avlonitis et al., "CD133+ cancer stem-like cells in small cell lung cancer are highly tumorigenic and chemoresistant but sensitive to a novel neuropeptide antagonist," Cancer Research, vol. 74, no. 5, pp. 1554-1565, 2014.

[89] N. Bora-Singhal, D. Perumal, J. Nguyen, and S. Chellappan, "Glil-mediated regulation of Sox2 facilitates self-renewal of stem-like cells and confers resistance to EGFR inhibitors in non-small cell lung cancer," Neoplasia, vol. 17, no. 7, pp. 538-551, 2015.

[90] W.-J. Chen, C.-C. Ho, Y.-L. Chang et al., "Cancer-associated fibroblasts regulate the plasticity of lung cancer stemness via paracrine signalling," Nature Communications, vol. 5, article 3472, 2014.

[91] P. Yuan, H. Kadara, C. Behrens et al., "Sex determining region Y-box 2 (SOX2) is a potential cell-lineage gene highly expressed in the pathogenesis of squamous cell carcinomas of the lung," PLoS ONE, vol. 5, no. 2, Article ID e9112, 2010.

[92] V. Justilien, M. P. Walsh, S. A. Ali, E. A. Thompson, N. R. Murray, and A. P. Fields, "The PRKCI and SOX2 Oncogenes are coamplified and cooperate to activate hedgehog signaling in lung squamous cell carcinoma," Cancer Cell, vol. 25, no. 2, pp. 139-151, 2014.

[93] C. Choe, Y.-S. Shin, C. Kim et al., "Crosstalk with cancerassociated fibroblasts induces resistance of non-small cell lung cancer cells to epidermal growth factor receptor tyrosine kinase inhibition," OncoTargets and Therapy, vol. 8, pp. 3665-3678, 2015.

[94] R. Katayama, A. T. Shaw, T. M. Khan et al., "Mechanisms of acquired crizotinib resistance in ALK-rearranged lung cancers," Science translational medicine, vol. 4, no. 120, Article ID 120ral7, 2012.

[95] S. Kobayashi, T. J. Boggon, T. Dayaram et al., "EGFR mutation and resistance of non-small-cell lung cancer to gefitinib," The New England Journal of Medicine, vol. 352, no. 8, pp. 786-792, 2005.

[96] S. Pan, X. Wu, J. Jiang et al., "Discovery of NVP-LDE225, a potent and selective smoothened antagonist," ACS Medicinal Chemistry Letters, vol. 1, no. 3, pp. 130-134, 2010.

[97] C. M. Della Corte, C. Bellevicine, G. Vicidomini et al., "SMO gene amplification and activation of the hedgehog pathway as novel mechanisms of resistance to anti-epidermal growth factor receptor drugs in human lung cancer," Clinical Cancer Research, vol. 21, no. 20, pp. 4686-4697, 2015. 
[98] K. D. Robarge, S. A. Brunton, G. M. Castanedo et al., "GDC0449-A potent inhibitor of the hedgehog pathway," Bioorganic and Medicinal Chemistry Letters, vol. 19, no. 19, pp. 5576-5581, 2009.

[99] M. Lauth, Å. Bergström, T. Shimokawa, and R. Toftgård, "Inhibition of GLI-mediated transcription and tumor cell growth by small-molecule antagonists," Proceedings of the National Academy of Sciences of the United States of America, vol. 104, no. 20, pp. 8455-8460, 2007.

[100] P. Infante, M. Mori, R. Alfonsi et al., "Gli1/DNA interaction is a druggable target for Hedgehog-dependent tumors," The EMBO Journal, vol. 34, no. 2, pp. 200-217, 2015.

[101] L. Zhang, L. Li, M. Jiao et al., "Genistein inhibits the stemness properties of prostate cancer cells through targeting HedgehogGlil pathway," Cancer Letters, vol. 323, no. 1, pp. 48-57, 2012.

[102] J. Kim, J. J. Lee, J. Kim, D. Gardner, and P. A. Beachy, "Arsenic antagonizes the Hedgehog pathway by preventing ciliary accumulation and reducing stability of the Gli2 transcriptional effector," Proceedings of the National Academy of Sciences of the United States of America, vol. 107, no. 30, pp. 13432-13437, 2010.

[103] K. Chang, M. Yang, J. Zheng et al., "Arsenic trioxide inhibits cancer stem-like cells via down-regulation of Glil in lung cancer," American Journal of Translational Research, vol. 8, no. 2, pp. 1133-1143, 2016. 

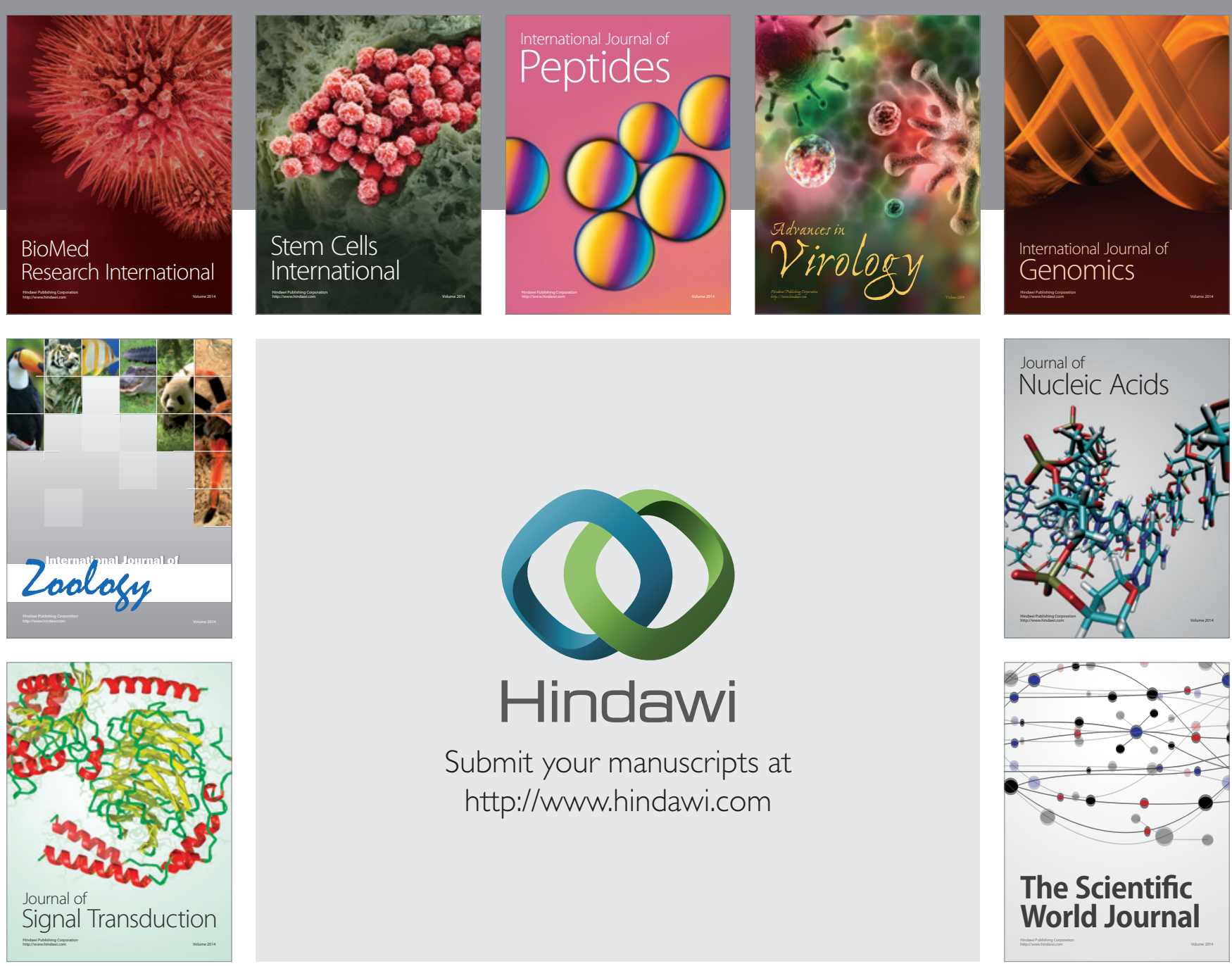

Submit your manuscripts at

http://www.hindawi.com
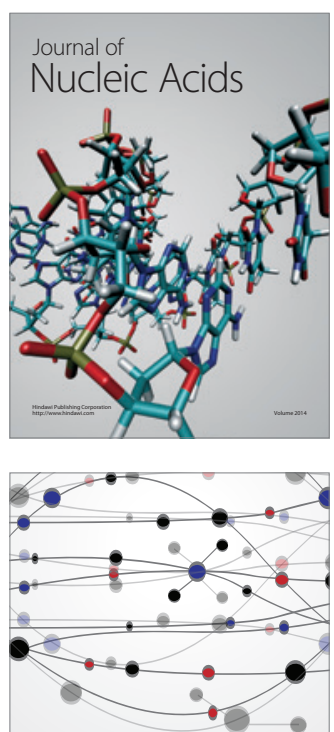

The Scientific World Journal
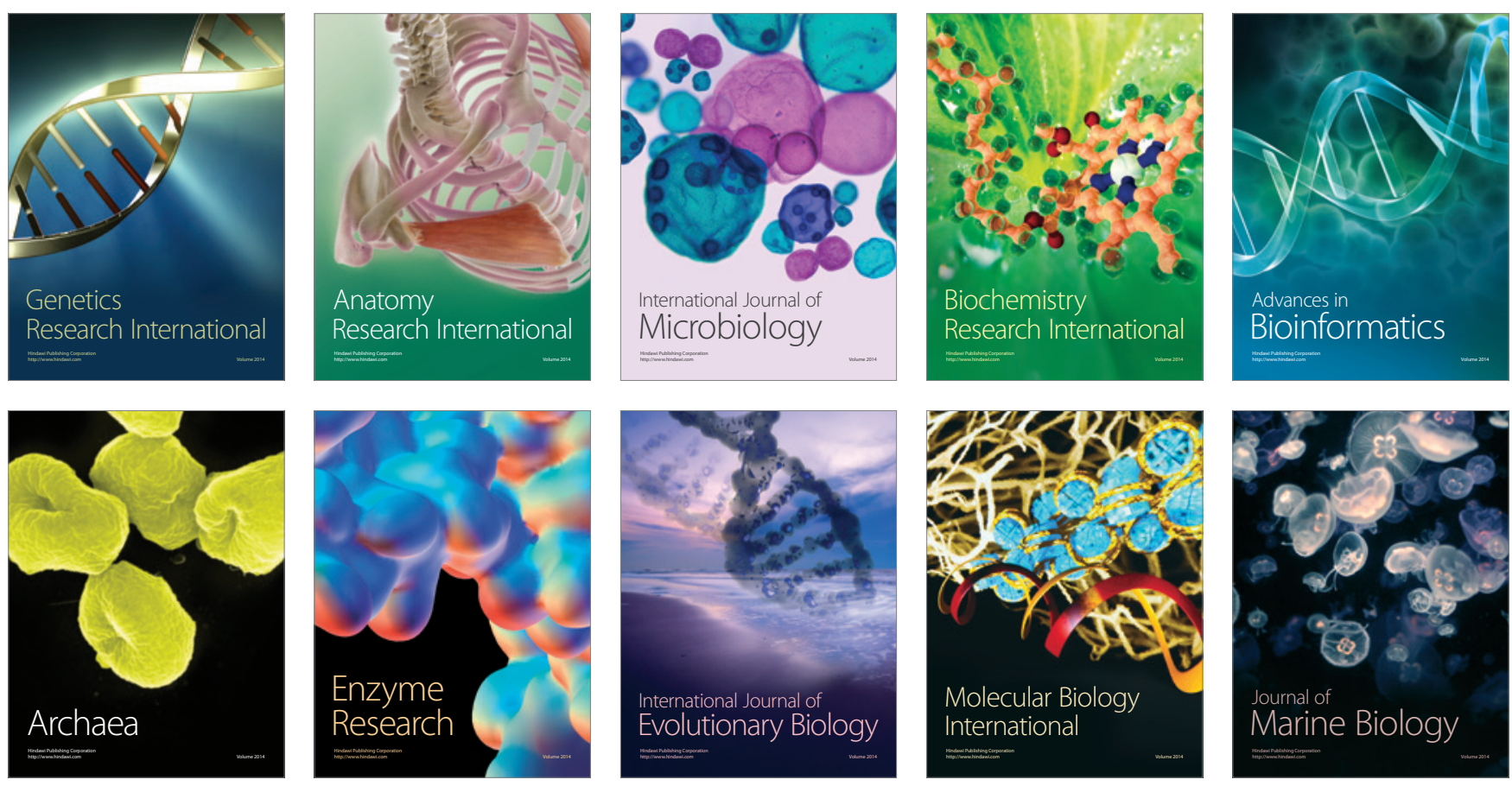